\title{
Comparative physical maps derived from BAC end sequences of tilapia (Oreochromis niloticus)
}

\author{
Lucile Soler ${ }^{1}$, Matthew A Conte ${ }^{2}$, Takayuki Katagiri ${ }^{3}$, Aimee E Howe ${ }^{2}$, Bo-Young Lee ${ }^{2}$, Chris Amemiya ${ }^{4}$, \\ Andrew Stuart ${ }^{4}$, Carole Dossat ${ }^{5}$, Julie Poulain ${ }^{5}$, Jeremy Johnson ${ }^{6}$, Federica Di Palma ${ }^{6}$, Kerstin Lindblad-Toh ${ }^{6}$, \\ Jean-Francois Baroiller ${ }^{1}$, Helena D'Cotta', Catherine Ozouf-Costaz ${ }^{7}$, Thomas D Kocher ${ }^{2^{*}}$
}

\begin{abstract}
Background: The Nile tilapia is the second most important fish in aquaculture. It is an excellent laboratory model, and is closely related to the African lake cichlids famous for their rapid rates of speciation. A suite of genomic resources has been developed for this species, including genetic maps and ESTs. Here we analyze BAC endsequences to develop comparative physical maps, and estimate the number of genome rearrangements, between tilapia and other model fish species.

Results: We obtained sequence from one or both ends of 106,259 tilapia BACs. BLAST analysis against the genome assemblies of stickleback, medaka and pufferfish allowed identification of homologies for approximately 25,000 BACs for each species. We calculate that rearrangement breakpoints between tilapia and these species occur about every $3 \mathrm{Mb}$ across the genome. Analysis of 35,000 clones previously assembled into contigs by restriction fingerprints allowed identification of longer-range syntenies.

Conclusions: Our data suggest that chromosomal evolution in recent teleosts is dominated by alternate loss of gene duplicates, and by intra-chromosomal rearrangements ( one per million years). These physical maps are a useful resource for comparative positional cloning of traits in cichlid fishes. The paired BAC end sequences from these clones will be an important resource for scaffolding forthcoming shotgun sequence assemblies of the tilapia genome.
\end{abstract}

\section{Background}

Tilapia (Oreochromis spp.) are among the most important species in aquaculture and a primary source of animal protein for millions of people in the developing world [1]. Only limited efforts have been made toward genetic improvement of these species [2]. The sequence of the tilapia genome will be a fundamental resource used for genetic selection, on traits such as growth performance and disease resistance, to create strains of fish optimized for the unique culture conditions of each country.

Tilapia and other closely related species of African cichlid fishes are also widely used in basic research. Because of their intimate physiological relationship with the environment, tilapia are ideal for studies of ion

\footnotetext{
* Correspondence: tdk@umd.edu

${ }^{2}$ Department of Biology, University of Maryland, College Park, Maryland 20742, USA

Full list of author information is available at the end of the article
}

regulation [3,4], the accumulation of heavy metals [5], and detoxification of biotoxins [6]. Nile tilapia expressing a humanized insulin gene are being studied as a source of islet cells which might be transplanted into humans for control of type I diabetes [7]. Tilapia are also an important model for studying environmental influences on sex differentiation [8]. The closely related haplochromine cichlids of the East African lakes are a model system for studying the genetic basis of behavior [9] and evolutionary processes of adaptation and speciation [10].

\section{Cichlid genomics}

Considerable progress has been made in developing genomic resources for tilapia and other East African cichlid fishes. Genetic maps have been published for tilapia [11], Lake Malawi haplochromines [12], and Astatotilapia burtoni [13]. There are also extensive collections of ESTs for Lake Victoria haplochromines

\section{() Biomed Central}


[14,15], A. burtoni $[16,17]$ and Nile tilapia [18]. Several BAC libraries have been constructed for Nile tilapia [19], and fingerprinted to construct a physical map [20]. BAC libraries have been constructed also for haplochromine cichlids from lakes Malawi [21], Victoria [22] and Tanganyika [23].

\section{Comparative physical maps}

Comparative maps have been a useful intermediate resource for many agricultural species before complete genome sequences were available [24-26]. Most often these comparative maps have relied on mapping homologous gene markers in radiation hybrid panels [27], but comparative maps have also been based on analysis of $\mathrm{BAC}$ end sequences $[28,29]$. Until a complete genome sequence is available for tilapia, comparative maps to the genome sequences of model fish species will provide the best organization of the partial sequence data for cichlid fishes.

The utility of a comparative map is proportional to the extent to which synteny exists between the two genomes. Useful comparative maps have been constructed between cattle and human (100MY divergence) [30]. The divergence among many fish lineages is much older, creating the potential for more extensive genome rearrangements. The Ostariophysi (e.g. zebrafish) and Acanthopterygii (e.g. medaka) diverged $\sim 300 \mathrm{MY}$ ago [31]. Divergence among Percomorph groups (e.g. Tilapia and pufferfish) occurred more than 100MY ago [32]. The utility of comparative maps across these greater evolutionary distances is not yet clear.

Early research suggested that the rate of chromosome evolution is relatively low in non-mammalian vertebrates [33]. Recently it has been suggested that the rate of chromosomal rearrangement increases immediately after episodes of whole-genome duplication [34]. Teleost fishes experienced an additional round of whole genome duplication about $300 \mathrm{MY}$ ago [35], and recent papers have suggested that fishes continue to have a high rate of chromosomal rearrangement [36]. However, the more extensive inter-chromosomal rearrangements detected in the zebrafish genome may be due to unique evolutionary processes in that lineage, and there appear to have been no major inter-chromosomal rearrangements in the medaka genome during the last 300MY [37]. The green pufferfish shows relatively little inter-chromosomal rearrangement since divergence from the ancestral bony vertebrate [38]. Most of the changes in the pufferfish lineage represent fusions that reduced the chromosome number after whole genome duplications.

The goal of the present study was to construct a comparative physical map between tilapia and the latest sequence assemblies for three other percomorph species: stickleback, medaka and pufferfish. From this comparative map we estimate the extent of chromosomal rearrangement during the recent evolution of these species.

\section{Results and Discussion \\ New BAC library}

The BAC library (VMRC-44) constructed at the Benaroya Research Institute consists of 73,728 clones (192 384-well plates) with an average insert size of 150 $\mathrm{kb}$. This represents a total of $11 \mathrm{Gbp}$ or approximately $10 \times$ coverage of the tilapia genome. The methods used to prepare this library are presented in Additional file 1.

\section{Sequencing statistics Genoscope}

The construction of the BAC libraries sequenced at Genoscope was reported previously [19]. A total of 35,000 clones from these libraries (average insert $182 \mathrm{~kb}, \sim 5.6 \times$ genome coverage), have been restriction fingerprinted and assembled into 3,600 contigs [20]. Genoscope end sequenced a total of 40,704 clones (52 plates from library 3 and 54 plates from library 4). From 37,383 clones, a total of 68,032 end sequences were obtained, representing $6.8 \times$ clone coverage of the genome. The mean trimmed length of the sequences was $562.6 \mathrm{bp}$, for a total dataset of $38,272,386$ bp representing $3.8 \%$ sequence coverage of the genome.

\section{Broad}

The Broad Institute end sequenced 73,728 clones (192 plates) from the Benaroya library, obtaining a sequence for at least one end of 68,876 clones, representing $10.0 \times$ clone coverage of the genome. Multiple attempts were made to sequence some clones and therefore, a total of 153,216 end sequences were finally submitted to GenBank. The mean length of the sequences was 757.3 $\mathrm{bp}$, for a dataset of $116,029,366 \mathrm{bp}$. After quality trimming and vector removal with Lucy, a total of 124,995 sequences remained, with a mean length of $527.3 \mathrm{bp}$, for a total of $65,912,624 \mathrm{bp}$, representing $6.6 \%$ sequence coverage of the genome. These sequences were previously analyzed for their repeat content [39].

\section{Microsatellites}

Microsatellite motifs were identified in 7,230 (3.7\%) of the 193,027 sequences. These included 5,027 dinucleotide, 1,250 trinucleotide, and 953 tetranucleotide repeats (Additional file 2Table S1). Over half of the repeats $(3,887)$ were AC dinucleotides. AT and AG dinucleotides were also abundant. AAT was the most frequent trinucleotide. These microsatellites could be exploited to develop new genetic markers and could be used to anchor the FPC-based physical map [20] to the genetic map [11]. 


\section{Genes}

A total of $16,636(8.6 \%)$ repeat-masked sequences had a significant (1e-5) BLASTx hit to the Uniprot database. We found that $38,020(19.7 \%)$ of the repeat-masked sequences had a significant (1e-50) BLASTn hit to the 116,899 Nile tilapia EST set [40]. Therefore, 49,823 (25.8\%) of the sequences had either a significant BLASTx hit to Uniprot or a significant BLASTn hit to the Nile tilapia ESTs. There were 4,833 (2.5\%) sequences that had a significant hit to both Uniprot and the Nile tilapia ESTs.

\section{Comparative mapping}

A total of 193,027 BAC end sequences were BLASTed against the genome assemblies of stickleback, medaka and pufferfish. The results are summarized in Table 1. The proportion of sequences that had hits with e-values less than $\mathrm{e}^{-10}$ ranged from 11 percent against pufferfish, 15 percent against medaka and 17 percent against stickleback. Twenty-eight percent of the BACs had at least one hit to the stickleback genome assembly.

We classified the BACs into one of four types, according to the pattern of BLAST hit. Type 1 clones are those for which only a single sequence produced a hit in the target genome. Type 2 clones are those in which the sequences from the two ends of the BAC hit in the appropriate opposing orientation within $300 \mathrm{~kb}$ in the target genome. Type 3 clones are those in which the two end sequences of a BAC hit the same chromosome in the target genome outside of the $300 \mathrm{~kb}$ range. Type 4 BACs are those in which the two sequences hit different chromosomes in the target genome.
Since the average BLAST hit rate against the stickleback genome is $17 \%$, we expected the proportion of clones with hits on both ends would be $2.9 \%$. In fact we observed a slightly greater proportion (3.7\%), possibly reflecting a clustering of conserved sequences in the genomes. When both ends of a BAC had BLAST hits, they were most often found within $300 \mathrm{~kb}$ on the same chromosome in the target genome (type 2). A much smaller proportion (3-5\%) were found at larger distances on the same chromosome in the target genome (type 3).

\section{Conservation of gene order}

We can use the ratios of type 2, 3 and 4 hits (Table 1) to estimate the number of rearrangements between genomes. Across the three species, $27-41 \%$ of double hit clones are type 3 or 4 . If the BAC clone inserts average $150 \mathrm{~kb}$, and every third clone has a break in synteny, it would suggest a breakpoint every $3 \times 150 \mathrm{~kb}=450 \mathrm{~kb}$ across the genome. This is equivalent to more than 2000 breakpoints across the genome, or about 100 breakpoints per chromosome. We suspect this simple statistic overestimates the true number of chromosomal rearrangements.

The best estimate of intra-chromosomal rearrangements is the number of type 3 BACs relative to the number of type $2+$ type 3 BACs. This proportion is between 3 and $6 \%$, suggesting an intra-chromosomal rearrangement every $20 \times 150 \mathrm{~kb}=3 \mathrm{Mb}$. If the average chromosome is $48 \mathrm{Mb}$, this suggests about 16 breakpoints (e.g. 8 inversions) per chromosome. We detected a mean of 2.1 breakpoints per chromosome, with at least one rearrangement on each stickleback chromosome (Additional file

Table 1 BLAST statistics against three fish genome assemblies

\begin{tabular}{|c|c|c|c|c|c|c|c|c|c|c|}
\hline & $\begin{array}{l}\text { \# BACs } \\
\text { one end }\end{array}$ & $\begin{array}{c}\text { \# BACs } \\
\text { both ends }\end{array}$ & $\begin{array}{c}\text { Sequences } \\
\text { w/hit }\end{array}$ & $\begin{array}{l}\text { BACs } \\
\text { w/hit }\end{array}$ & Type $1^{*}$ & Type $2^{*}$ & Type $3 *$ & Type $4^{*}$ & $3 /(2+3)$ & $\begin{array}{c}(3+4) / \\
(2+3+4)\end{array}$ \\
\hline \multicolumn{11}{|l|}{ Stickleback } \\
\hline Genoscope & 6,734 & 30,649 & 11,229 & 10,048 & 7,987 & 797 & 54 & 153 & 0.063 & 0.206 \\
\hline Broad & 12,758 & 56,118 & 21,754 & 19,510 & 14,405 & 1,416 & 142 & 633 & 0.054 & 0.286 \\
\hline Combined & 19,492 & 86,767 & 33,053 & 29,558 & 22,392 & 2,213 & 196 & 786 & 0.057 & 0.259 \\
\hline \multicolumn{11}{|l|}{ Medaka } \\
\hline Genoscope & 6,734 & 30,649 & 9,764 & 9,278 & 7,087 & 469 & 43 & 226 & 0.084 & 0.364 \\
\hline Broad & 12,758 & 56,118 & 19,699 & 17,943 & 13,907 & 886 & 51 & 624 & 0.054 & 0.432 \\
\hline Combined & 19,492 & 86,767 & 29,463 & 27,221 & 20,994 & 1,355 & 94 & 850 & 0.065 & 0.410 \\
\hline \multicolumn{11}{|l|}{ Tetraodon } \\
\hline Genoscope & 6,734 & 30,649 & 6,931 & 6,386 & 3,879 & 265 & 14 & 62 & 0.050 & 0.222 \\
\hline Broad & 12,758 & 56,118 & 14,260 & 13,227 & 7,279 & 503 & 19 & 188 & 0.036 & 0.291 \\
\hline Combined & 19,492 & 86,767 & 21,191 & 19,613 & 11,158 & 768 & 33 & 250 & 0.041 & 0.269 \\
\hline
\end{tabular}

*Numbers for type 1, 2, 3 \& 4 do not include hits to the contigs in the 'unordered chromosome' of each genome assembly. Type 2 hits were scored when the two end sequences of a clone hit within $300 \mathrm{~kb}$ of each other in the target genome. 
3Table S2). The observed breakpoints were spanned by an average of 3.5 BAC clones. Unfortunately, the relatively low clone coverage of the type $3 \mathrm{BACs}$ does not allow us to identify all of the likely breakpoints, or precisely map their locations. Still, the high end of these estimates (8 inversions/chromosome) suggests there have been only 160 inversions since the divergence of tilapia and stickleback. The type 3 hits are visualized in Circos plots in Additional files 4, 5, 6, Figures S1-S3.

Type 4 BACs are possible evidence of inter-chromosomal rearrangements, and represent $24-37 \%$ of the two-hit BACs. This might suggest more than 100 breakpoints in synteny for each chromosome. However, we do not think this statistic is an indication of a large number of interchromosomal transfers of genes. Rather, it probably includes many instances in which one of the BLAST matches is to a paralog on a second chromosome. For example, if the syntenic copy of the gene has been lost, BLAST will identify a paralog on another chromosome as the best hit. This kind of gene loss is a common feature of fish genomes, which underwent a whole-genome duplication about $300 \mathrm{MY}$ ago. Alternate loss of even a small proportion of genes from these duplicated regions would be sufficient to create the pattern. There are about 1,250 genes/chromosome, and if only $5 \%$ of them ( 60 genes/chromosome) were deleted after the whole genome duplication, it would be sufficient to create the pattern we see in the BAC data. The fact that type 4 BLAST hits have much lower e-values than type 2 BLAST hits (Figure 1) tends to reinforce this view.

We mapped the rearrangements onto a phylogeny of the four species. The results suggest that approximately 15-20 rearrangements have occurred on each lineage since they diverged from their common ancestor. There is no indication that the rate of rearrangement is higher in one lineage than another.

\section{Comparative physical maps}

These BLAST results are displayed in a GBrowse interface at http://www.BouillaBase.org (Figure 2). Separate tracks display the type 1, 2, 3 and 4 BLAST hits. An additional track displays the BLAST hits from each of the fingerprint contigs in the previous physical map [20]. Because these FPC contigs contain multiple BAC clones, they help to tie the physical map together at larger scales than the end sequences of individual clones.

\section{Conclusions}

End-sequencing of these BAC libraries was a key step in preparing the tilapia genome for shotgun sequencing. Together with the BAC fingerprint database, these sequences will provide long-range structure for

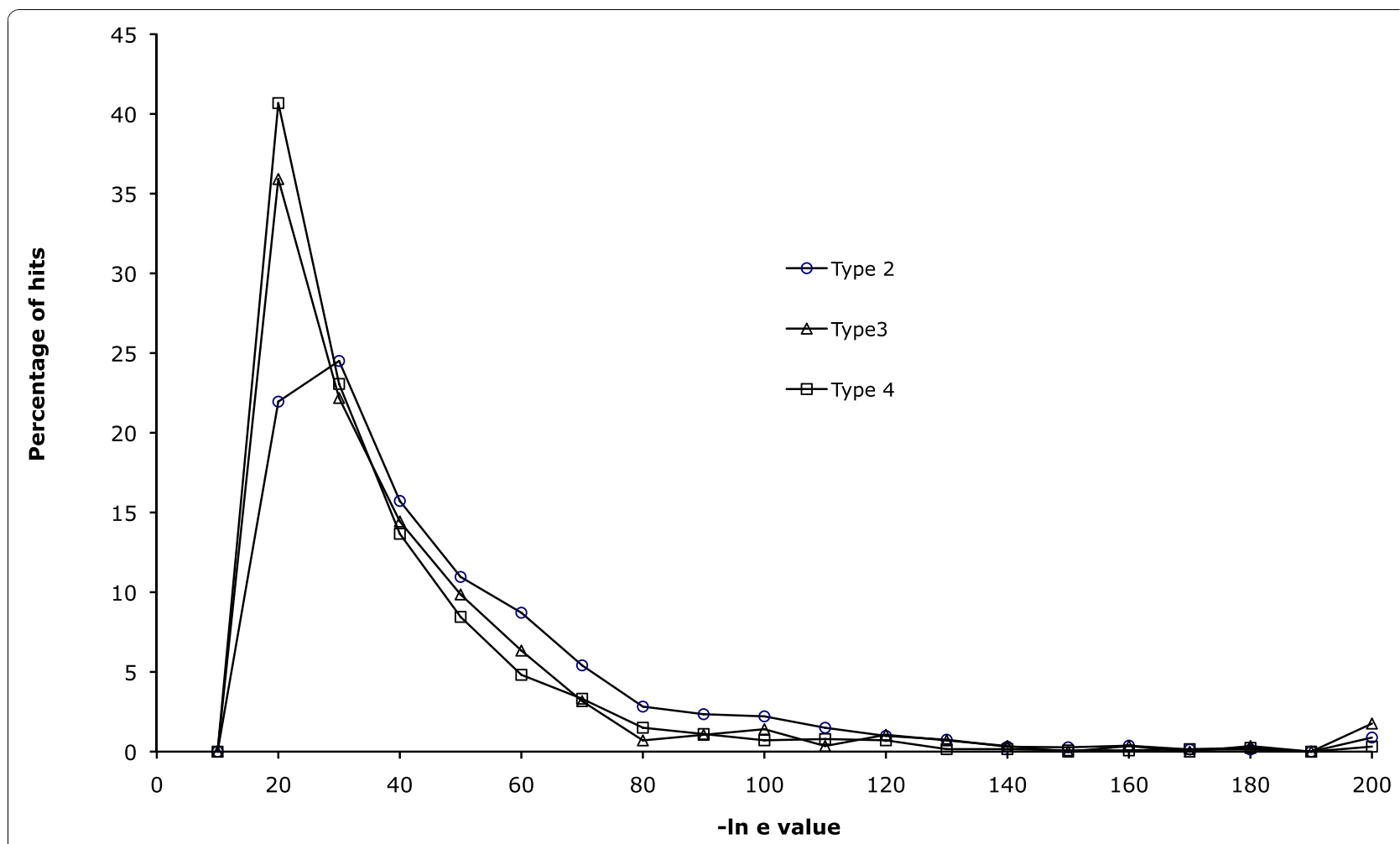

Figure 1 Distribution of BLAST scores for type 2, 3 and 4 hits of the Broad Institute tilapia BAC end sequences on the stickleback genome assembly. The distribution was truncated at $\mathrm{e}^{-10}$. 


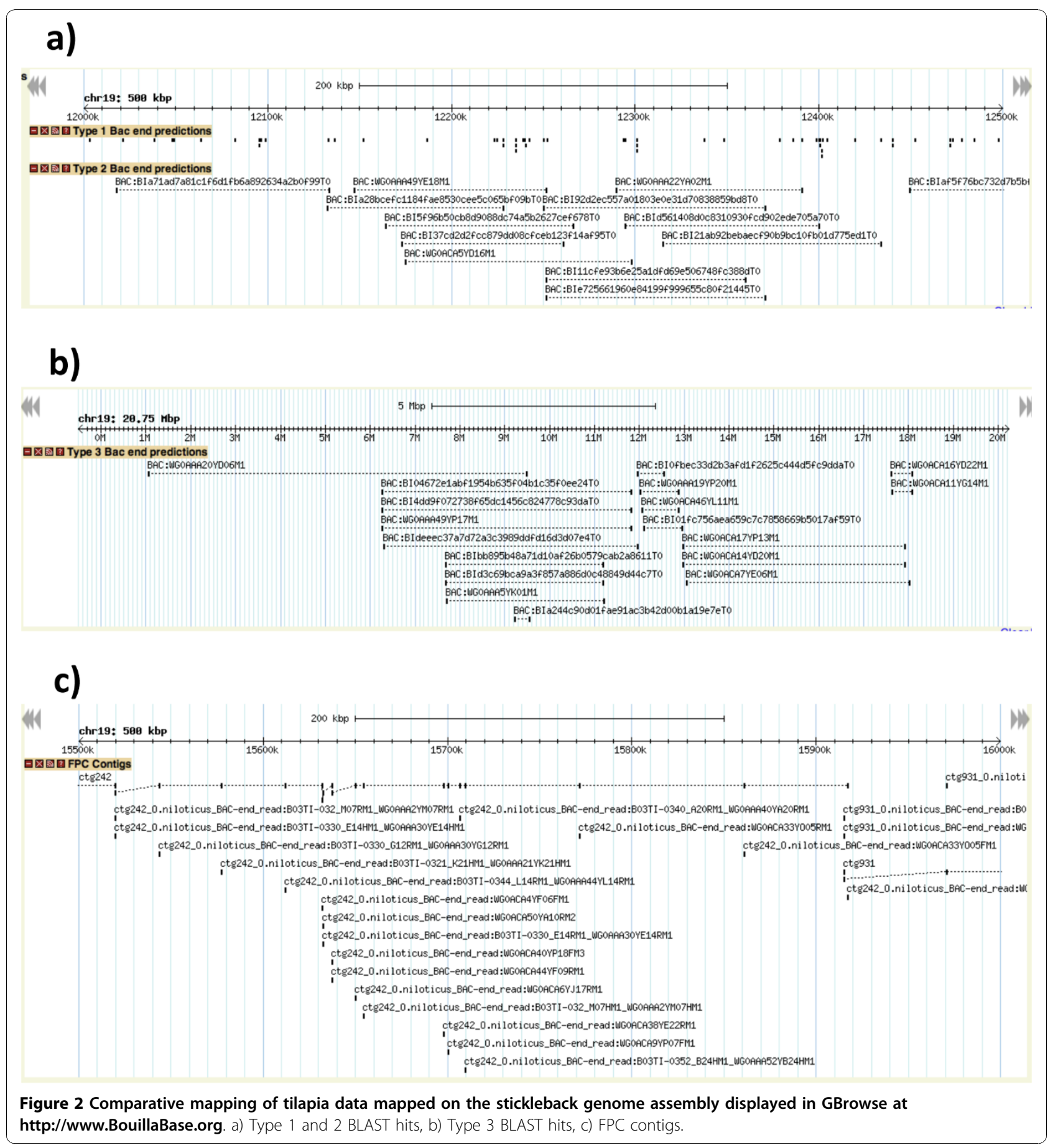

scaffolding the contigs of genome assemblies to construct a golden path across the genome.

Recent molecular phylogenies appear to have reached a consensus that cichlids are more closely related to medaka than to either pufferfish or stickleback [40-42]. Nevertheless, a higher number of the tilapia BAC end sequences hit stickleback $(33,053)$ than either medaka $(29,463)$ or pufferfish $(21,191)$. This discrepancy might be due to variation in the quality of each assembly, or it might support an alternative phylogenetic reconstruction. Regardless, it appears that the stickleback sequence is currently the best reference sequence for building comparative maps of tilapia [43].

Finally, these data suggest that chromosomal evolution in recent teleosts is dominated by alternate loss of gene duplicates, and by intra-chromosomal rearrangements. 
The rate of these rearrangements is relatively slow, on the order of one per million years. So the prospects are good for building useful comparative maps between sequenced genomes and the large number of as yet unsequenced teleost species of commercial or scientific importance.

\section{Methods}

\section{Sequence trimming}

Both trimmed and untrimmed quality scores and FASTA sequences for the Genoscope library 4 sequences were available, whereas only trimmed FASTA sequences for the Genoscope library 3 were available. To achieve essentially the same level of trimming for both Genoscope libraries and the Broad library, the Genoscope library 4 data was used to determine a set of parameters that trimmed the data in the same way as had been done for both the Genoscope libraries. The following Lucy 1.20p [44] settings were used: -error 0.0250 .02 , -bracket 10 0.005 , -window 500.08100 .12 , and -vector with the FASTA sequence of the pBAC-Lac cloning vector [45] for the Genoscope libraries and the FASTA sequence of the pCC1BAC cloning vector (Epicentre Biotechnologies) for the Broad library.

\section{Annotation}

\section{Identification of microsatellites}

We scanned the BAC end sequences for microsatellites that might be useful for genetic mapping. We used the Tandem Repeats Finder http://tandem.bu.edu/trf/trf. html[46] to identify microsatellite motifs. The BAC ends containing microsatellites have been color-coded in the annotation tracks in the GMOD browser.

\section{Identification of genes}

The BAC end sequences were masked with RepeatMasker version open-3.2.8 [47] against a combination of the Repbase [48] RepeatMasker libraries, release 20090604 and tilapia specific repeats [39]. The sequences were then aligned to the Uniprot database (release-2010_05) using BLASTx, and a database of 116,899 Sanger ESTs from Nile tilapia [18] using BLASTn. Significant hits were defined with an e-value threshold of $1 \mathrm{e}^{-5}$ for Uniprot, or $1 \mathrm{e}^{-50}$ for the ESTs.

\section{BLAST analysis}

Comparative mapping was performed by running BLASTn against the pufferfish, stickleback and medaka genome assemblies. The genomes were downloaded from the UCSC Genome Browser http://hgdownload.cse.ucsc. edu/downloads.html. The following versions were used for the respective genomes: Feb. 2004 (Genoscope 7/tetNig1), Feb. 2006 (Broad/gasAcu1), and Oct. 2005 (NIG/ UT MEDAKA1/oryLat2). FASTA sequences were downloaded and formatted into BLAST databases for use with the NCBI BLASTall tool and scripts utilizing BioPerl were used to parse the results. Type 2 hits were defined as mate pairs that hit the target genome in opposing orientation at a distance of $300 \mathrm{~kb}$ or less. Type 3 hits were defined as mate pairs that hit the same chromosome, regardless of orientation. Type 4 hits were defined as mate pairs that hit different chromosomes. The positions of the BLAST hits were visualized with Circos [49].

\section{Online access to the resource}

We used the GMOD browser http://www.gmod.org to develop a comparative genome server for fishes that maps tilapia ESTs and BAC end-sequences onto the genome assemblies of stickleback, medaka and pufferfish. This server can be accessed through our www site http://www.BouillaBase.org.

\section{Additional data files}

The Benaroya/Broad BAC end sequences are available in the NCBI Trace Archive under Center_Project 'G1447'. The Genoscope sequences are available as accession numbers FQ242537 - FQ280267.

\section{Additional material}

Additional file 1: Supplemental Methods. Description of methods used in constructing the BAC library.

Additional file 2: Table S1 Microsatellite motifs identified in the BAC end sequences.

Additional file 3: Table S2 Number of type 3 BACs spanning potential recombination breakpoints in the comparative map to stickleback.

Additional file 4: Figure S1 Circos plot of the type 3 BLAST hits on the stickleback genome. The chromosomes of the stickleback genome are represented on the circle. The position of BAC mate pair BLAST hits are indicated with red arcs.

Additional file 5: Figure S2 Circos plot of the type 3 BLAST hits on the medaka genome. The chromosomes of the medaka genome are represented on the circle. The position of BAC mate pair BLAST hits are indicated with red arcs.

Additional file 6: Figure S3 Circos plot of the type 3 BLAST hits on the Tetraodon genome. The chromosomes of the Tetraodon genome are represented on the circle. The position of BAC mate pair BLAST hits are indicated with red arcs.

\section{Acknowledgements}

This work was supported by a grant from the USDA-NRICGP (\#2006-04830) to TDK. BAC end-sequences from Genoscope have been produced through the Project "BAC end-sequencing for comparative genomics and assembly of the genome of tilapia Oreochromis niloticus" funded by CNS. We thank Elodie Pepey for her help with the robotics at CIRAD. The authors would like to thank Gaëtan Droc from the Cirad joint unit UMR DAP (Plant Development and Genetic Improvement) for help with the Circos diagrams. Thanks also to the Broad Institute Sequencing Platform for sequencing the Benaroya/Broad BAC library and making the data available.

\section{Author details}

'CIRAD-PERSYST, Aquaculture Research Unit, TA B-20/A, Campus

International de Baillarguet, 34398 Montpellier cedex 5, France. ${ }^{2}$ Department 
of Biology, University of Maryland, College Park, Maryland 20742, USA ${ }^{3}$ Laboratory of Fish Health Management, Tokyo University of Marine Science and Technology, 4-5-7 Konan, Minato-Ku Tokyo, 108-8477, Japan. ${ }^{4}$ Genome Resource Center, Benaroya Research Institute at Virginia Mason, 1201 Ninth Avenue, Seattle, WA 98101 USA. ${ }^{5}$ CEA, DSV, Genoscope, 2 rue Gaston Crémieux, CP5706 91057 Evry cedex, France. ${ }^{6}$ Broad Institute, 7 Cambridge Center, Cambridge, Massachusetts 02142, USA. ${ }^{7}$ CNRS UMR 7138 « Systématique, Evolution, Adaptation », MNHN Département Systématique et Evolution, C.P. 26, 57 rue Cuvier 75231 PARIS Cedex 05, France.

\section{Authors' contributions}

LS and MAC carried out the bioinformatic analyses. TK, AEH and BYL constructed and prepared the BAC libraries for sequencing at Genoscope. CA and AS constructed the BAC library that was sequenced at the Broad Institute. CD and JP sequenced the BAC libraries at Genoscope. JJ, FDP and $\mathrm{KLT}$ organized the sequencing at the Broad Institute. JFB, HDC, COC and TDK prepared the manuscript. All authors read and approved the final manuscript.

Received: 13 June 2010 Accepted: 16 November 2010 Published: 16 November 2010

\section{References}

1. Coward K, Little DC: Culture of the 'aquatic chicken': present concerns and future prospects. Biologist (London) 2001, 48:12-6.

2. Hulata G: Genetic manipulations in aquaculture: a review of stock improvement by classical and modern technologies. Genetica 2001, 111:155-73.

3. Fiol DF, Chan SY, Kültz D: Regulation of osmotic stress transcription factor 1 (Ostf1) in tilapia (Oreochromis mossambicus) gill epithelium during salinity stress. J Exp Biol 2006, 209:3257-65.

4. Breves JP, Hasegawa S, Yoshioka M, Fox BK, Davis LK, Lerner DT, Takei Y, Hirano T, Grau EG: Acute salinity challenges in Mozambique and Nile tilapia: Differential responses of plasma prolactin, growth hormone and branchial expression of ion transporters. Gen Comp Endocrinol 2010, 167:135-142.

5. Wang F, Leung AO, Wu SC, Yang MS, Wong MH: Chemical and ecotoxicological analyses of sediments and elutriates of contaminated rivers due to e-waste recycling activities using a diverse battery of bioassays. Environ Pollut 2009, 157:2082-90.

6. Prieto Al, Jos A, Pichardo S, Moreno I, de Sotomayor MA, Moyano R, Blanco A, Cameán AM: Time-dependent protective efficacy of Trolox (vitamin $\mathrm{E}$ analog) against microcystin-induced toxicity in tilapia (Oreochromis niloticus). Environ Toxicol 2009, 24:563-79.

7. Wright JR Jr, Pohajdak B: Cell therapy for diabetes using piscine islet tissue. Cell Transplant 2001, 10:125-43.

8. Baroiller JF, D'Cotta H, Saillant E: Environmental effects on fish sex determination and differentiation. Sex Dev 2009, 3:118-35.

9. Robinson GE, Fernald RD, Clayton DF: Genes and social behavior. Science 2008, 322:896-900

10. Kocher TD: Adaptive evolution and explosive speciation: the cichlid fish model. Nat Rev Genet 2004, 5:288-98.

11. Lee BY, Lee WJ, Streelman JT, Carleton KL, Howe AE, Hulata G, Slettan A Stern JE, Terai $Y$, Kocher TD: A second-generation genetic linkage map of tilapia (Oreochromis spp.). Genetics 2005, 170:237-44

12. Albertson RC, Streelman JT, Kocher TD: Directional selection has shaped the oral jaws of Lake Malawi cichlid fishes. Proc Natl Acad Sci USA 2003, 100:5252-7.

13. Sanetra M, Henning F, Fukamachi S, Meyer A: A microsatellite-based genetic linkage map of the cichlid fish, Astatotilapia burtoni (Teleostei): a comparison of genomic architectures among rapidly speciating cichlids. Genetics 2009, 182:387-97.

14. Watanabe M, Kobayashi N, Shin-i T, Horiike T, Tateno Y, Kohara Y, Okada N: Extensive analysis of ORF sequences from two different cichlid species in Lake Victoria provides molecular evidence for a recent radiation event of the Victoria species flock: identity of EST sequences between Haplochromis chilotes and Haplochromis sp. "Redtailsheller". Gene 2004, 343:263-9.

15. Kobayashi N, Watanabe M, Horiike T, Kohara Y, Okada N: Extensive analysis of EST sequences reveals that all cichlid species in Lake Victoria share almost identical transcript sets. Gene 2009, 441:187-91.
16. Renn SC, Aubin-Horth N, Hofmann HA: Biologically meaningful expression profiling across species using heterologous hybridization to a CDNA microarray. BMC Genomics 2004, 5:42.

17. Salzburger W, Renn SC, Steinke D, Braasch I, Hofmann HA, Meyer A: Annotation of expressed sequence tags for the East African cichlid fish Astatotilapia burtoni and evolutionary analyses of cichlid ORFs. BMC Genomics 2008, 9:96.

18. Lee BY, Howe AE, Conte MA, D'Cotta H, Pepey E, Baroiller JF, di Palma F, Carleton KL, Kocher TD: An EST resource for tilapia based on 17 normalized libraries and assembly of 116,899 sequence tags. BMC Genomics 2010, 11:278.

19. Katagiri T, Asakawa S, Minagawa S, Shimizu N, Hirono I, Aoki T: Construction and characterization of BAC libraries for three fish species; rainbow trout, carp and tilapia. Anim Genet 2001, 32:200-4.

20. Katagiri T, Kidd C, Tomasino E, Davis JT, Wishon C, Stern JE, Carleton KL, Howe AE, Kocher TD: A BAC-based physical map of the Nile tilapia genome. BMC Genomics 2005, 6:89.

21. Di Palma F, Kidd C, Borowsky R, Kocher TD: Construction of bacterial artificial chromosome libraries for the Lake Malawi cichlid (Metriaclima zebra), and the blind cavefish (Astyanax mexicanus). Zebrafish 2007, 4:41-7.

22. Watanabe M, Kobayashi N, Fujiyama A, Okada N: Construction of a BAC library for Haplochromis chilotes, a cichlid fish from Lake Victoria. Genes Genet Syst 2003, 78:103-5.

23. Lang M, Miyake T, Braasch I, Tinnemore D, Siegel N, Salzburger W, Amemiya CT, Meyer A: A BAC library of the East African haplochromine cichlid fish Astatotilapia burtoni. J Exp Zool B Mol Dev Evol 2006, 306:35-44.

24. Everts-van der Wind A, Larkin DM, Green CA, Elliott JS, Olmstead CA, Chiu R, Schein JE, Marra MA, Womack JE, Lewin HA: A high-resolution whole-genome cattle-human comparative map reveals details of mammalian chromosome evolution. Proc Natl Acad Sci USA 2005, 102:18526-31.

25. Dalrymple BP, Kirkness EF, Nefedov M, McWilliam S, Ratnakumar A, Barris W, Zhao S, Shetty J, Maddox JF, O'Grady M, Nicholas F, Crawford AM, Smith T, de Jong PJ, McEwan J, Oddy VH, Cockett NE, the International Sheep Genomics Consortium: Using comparative genomics to reorder the human genome sequence into a virtual sheep genome. Genome Biol 2007, 8:R152.

26. Reed KM, Chaves LD, Mendoza KM: An integrated and comparative genetic map of the turkey genome. Cytogenet Genome Res 2007, 119:113-26.

27. Raudsepp T, Gustafson-Seabury A, Durkin K, Wagner ML, Goh G, Seabury CM, Brinkmeyer-Langford C, Lee EJ, Agarwala R, Stallknecht-Rice E, Schäffer AA, Skow LC, Tozaki T, Yasue H, Penedo MC, Lyons LA, Khazanehdari KA, Binns MM, MacLeod JN, Distl O, Guérin G, Leeb T, Mickelson JR, Chowdhary BP: A 4,103 marker integrated physical and comparative map of the horse genome. Cytogenet Genome Res 2008, 122:28-36.

28. Rogatcheva MB, Chen K, Larkin DM, Meyers SN, Marron BM, He W, Schook LB, Beever JE: Piggy-BACing the human genome I: constructing a porcine BAC physical map through comparative genomics. Anim Biotechnol 2008, 19:28-42.

29. Liu H, Jiang Y, Wang S, Ninwichian P, Somridhivej B, Xu P, Abernathy J, Kucuktas H, Liu Z: Comparative analysis of catfish BAC end sequences with the zebrafish genome. BMC Genomics 2009, 10:592.

30. Larkin DM, Everts-van der Wind A, Rebeiz M, Schweitzer PA, Bachman S, Green C, Wright CL, Campos EJ, Benson LD, Edwards J, Liu L, Osoegawa K, Womack JE, de Jong PJ, Lewin HA: A cattle-human comparative map built with cattle BAC-ends and human genome sequence. Genome Res 2003, 13:1966-72.

31. Peng Z, Diogo R, He S: Teleost fishes (Teleostei). In The Timetree of Life. Edited by: Hedges SB, Kumar S. Oxford: Oxford University Press; 2009:335-338.

32. Santini F, Harmon L, Carnevale G, Alfaro ME: Did genome duplication drive the origin of teleosts? A comparative study of diversification in ray-finned fishes. BMC Evol Biol 2009, 9:194.

33. Wilson AC, Sarich VM, Maxson LR: The importance of gene rearrangement in evolution: evidence from studies on rates of chromosomal, protein, and anatomical evolution. Proc Natl Acad Sci USA 1974, 71:3028-3030.

34. Sémon M, Wolfe $\mathrm{KH}$ : Rearrangement rate following the whole-genome duplication in teleosts. Mol Biol Evol 2007, 24:860-7. 
35. Hurley IA, Mueller RL, Dunn KA, Schmidt EJ, Friedman M, Ho RK, Prince VE, Yang Z, Thomas MG, Coates Ml: A new time-scale for ray-finned fish evolution. Proc R SOC B 2007, 274:489-98.

36. Ravi $V$, Venkatesh B: Rapidly evolving fish genomes and teleost diversity. Curr Opin Genet Dev 2008, 18:544-50.

37. Kasahara M, Naruse K, Sasaki S, Nakatani Y, Qu W, Ahsan B, Yamada T, Nagayasu Y, Doi K, Kasai Y, Jindo T, Kobayashi D, Shimada A, Toyoda A, Kuroki Y, Fujiyama A, Sasaki T, Shimizu A, Asakawa S, Shimizu N, Hashimoto S, Yang J, Lee Y, Matsushima K, Sugano S, Sakaizumi M, Narita T, Ohishi K, Haga S, Ohta F, Nomoto H, Nogata K, Morishita T, Endo T, Shin-I T, Takeda H, Morishita S, Kohara Y: The medaka draft genome and insights into vertebrate genome evolution. Nature 2007, 447:714-9.

38. Jaillon O, Aury JM, Brunet F, Petit JL, Stange-Thomann N, Mauceli E, Bouneau L, Fischer C, Ozouf-Costaz C, Bernot A, Nicaud S, Jaffe D, Fisher S, Lutfalla G, Dossat C, Segurens B, Dasilva C, Salanoubat M, Levy M, Boudet N, Castellano S, Anthouard V, Jubin C, Castelli V, Katinka M, Vacherie B, Biémont C, Skalli Z, Cattolico L, Poulain J, De Berardinis V, Cruaud C, Duprat S, Brottier P, Coutanceau JP, Gouzy J, Parra G, Lardier G, Chapple C, McKernan K, McEwan P, Bosak S, Kellis M, Volff JN, Guigó R, Zody MC, Mesirov J, Lindblad-Toh K, Birren B, Nusbaum C, Kahn D, RobinsonRechavi M, Laudet V, Schachter V, Quétier F, Saurin W, Scarpelli C, Wincker P, Lander ES, Weissenbach J, Roest Crollius H: Genome duplication in the teleost fish Tetraodon nigroviridis reveals the early vertebrate proto-karyotype. Nature 2004, 431:946-57.

39. Shirak A, Grabherr M, Di Palma F, Lindblad-Toh K, Hulata G, Ron M, Kocher TD, Seroussi E: Identification of repetitive elements in the genome of Oreochromis niloticus: tilapia repeat masker. Mar Biotechnol (NY) 2010, 12:121-5.

40. Azuma Y, Kumazawa Y, Miya M, Mabuchi K, Nishida M: Mitogenomic evaluation of the historical biogeography of cichlids toward reliable dating of teleostean divergences. BMC Evol Biol 2008, 8:215.

41. Li B, Dettaï A, Cruaud C, Couloux A, Desoutter-Meniger M, Lecointre G: RNF213, a new nuclear marker for acanthomorph phylogeny. Mol Phylogenet Evol 2009, 50:345-63.

42. Chen W-J, Mayden RL: A phylogenomic perspective on the new era of ichthyology. Bioscience 2010, 60:421-432.

43. Sarropoulou E, Nousdili A, Magoulas A, Kotoulas G: Linking the genomes of nonmodel teleosts through comparative genomics. Mar Biotechnol 2008, 10:227-233.

44. Chou HH, Holmes MH: DNA sequence quality trimming and vector removal. Bioinformatics 2001, 17:1093-104.

45. Asakawa S, Abe I, Kudoh Y, Kishi N, Wang Y, Kubota R, Kudoh J, Kawasaki K, Minoshima S, Shimizu N: Human BAC library: construction and rapid screening. Gene 1997, 191:69-79.

46. Benson G: Tandem Repeats Finder: a program to analyze DNA sequences. Nucleic Acids Res 1999, 27:573-80.

47. Smit AFA, Hubley R, Green P: RepeatMasker Open-3.0. 1996-2010.[http:// www.repeatmasker.org].

48. Jurka J, Kapitonov W, Pavlicek A, Klonowski P, Kohany O, Walichiewicz J: Repbase Update, a database of eukaryotic repetitive elements. Cytogen Genome Res 2005, 110:462-467.

49. Krzywinski M, Schein J, Birol I, Connors J, Gascoyne R, Horsman D, Jones SJ, Marra MA: Circos: an information aesthetic for comparative genomics. Genome Res 2009, 19:1639-45.

doi:10.1186/1471-2164-11-636

Cite this article as: Soler et al.: Comparative physical maps derived from BAC end sequences of tilapia (Oreochromis niloticus). BMC Genomics $201011: 636$

\section{Submit your next manuscript to BioMed Central and take full advantage of:}

- Convenient online submission

- Thorough peer review

- No space constraints or color figure charges

- Immediate publication on acceptance

- Inclusion in PubMed, CAS, Scopus and Google Scholar

- Research which is freely available for redistribution

Submit your manuscript at www.biomedcentral.com/submit
Biomed Central 